\title{
Surface-enhanced Raman and DFT study on zidovudine
}

\author{
Nicoleta Elena Mircescu, Andrada Varvescu, Krisztian Herman, Vasile Chiş and \\ Nicolae Leopold* \\ Faculty of Physics, Babeş-Bolyai University, Cluj-Napoca, Romania
}

\begin{abstract}
This work presents a surface-enhanced Raman scattering (SERS) and density functional theory (DFT) study on neutral and deprotonated molecular forms of zidovudine. A $\mathrm{p} K_{\mathrm{a}}$ value of 9.55 was found for this compound, the deprotonation occurring at the imide nitrogen of the pyrimidine ring. The characteristic bands of the SERS spectra were assigned considering both, the calculated vibrational wavenumbers and Raman intensity pattern. The adsorption geometry on the silver surface of zidovudine was derived based on the molecular electrostatic potential (MEP) and the selective Raman bands enhancement.
\end{abstract}

Keywords: SERS, DFT, pH-dependence, zidovudine

\section{Introduction}

Zidovudine, $3^{\prime}$-azido- $3^{\prime}$-deoxythymidine (AZT), is an organic compound used to delay development of acquired immunodeficiency syndrome (AIDS) in patients infected with HIV, to reduce mother-tochild HIV transmission and as radiosensitiser in cancer radiotherapy, because it is a telomerase inhibitor with potential anticancer properties [14]. In order to avoid toxicity and also to achieve optimal drug concentration for viral suppression, it is now highly recommended a careful monitoring of drug levels [10].

In spite of its importance for pharmaceutical purposes, however, the reported spectroscopical studies on zidovudine are scarce. Cheng et al. [2] investigated different conformers of AZT by simulating their IR spectra. Improper hydrogen bonds caused blueshift in $\mathrm{C}-\mathrm{H}$ bond stretch vibrations in three conformers of zidovudine that have been studied using DFT in gas phase and in aqueous solution. Recently, Peepliwal et al. [9] developed and then validated two analytical methods for the quantification of AZT containing solid dosage form by using FTIR and UV-Vis spectroscopies. AZT showed no interference from the common additives and excipients, demonstrating that the recommended procedure is well suited for the assay and evaluation of drugs in pharmaceutical preparations. Nayak et al. [7] studied the sustained release of AZT using chitosan as a polymer matrix by applying IR spectroscopy and differential scanning calorimetry (DSC). They have demonstrated the stability of the drug during the encapsulation process and have shown more than $95 \%$ drug release in $12 \mathrm{~h}$. Rivas et al. [12,13] used normal Raman as well as SERS to carry out a structural study of AZT in solid state, aqueous solution and when adsorbed on silver nanoparticles prepared by reduction with citrate. Their study was focused

\footnotetext{
* Corresponding author: Nicolae Leopold, Faculty of Physics, Babeş-Bolyai University, Kogălniceanu 1, 400084 ClujNapoca, Romania. Tel.: +40 264 405300; Fax: +40 264 591906; E-mail: nicolae.leopold@ phys.ubbcluj.ro; URL: http://www. phys.ubbcluj.ro/ nicolae.leopold.
} 
on the effect of sugar substituents in the tautomerisation of thymine residue as well as on the conformational rearrangement of the molecule when adsorbed on the above metal surface. Raviolo et al. [11] investigated X-ray crystal and molecular structure, NMR, IR and Raman spectra, the thermal behavior of a novel carbonate of AZT and its lipophilic properties in order to enhance pharmacological effects. A study concerning the biodegradation of AZT in the environment was carried out by Kruszewska et al. [4] by using chromatoghraphic methods, mass analyses, NMR and vibrational methods.

In this study, SERS and DFT calculations were used for a proper investigation of the structure and electronic properties of AZT, with the particular aim of evidencing the characteristic SERS bands of this compound in its neutral and deprotonated forms.

\section{Experimental details}

SERS spectra were recorded using an Advantage 532 spectrometer (DeltaNu, USA), equipped with a frequency doubled NdYAG laser emitting at $532 \mathrm{~nm}$.

For SERS measurements, a silver colloidal SERS substrate was prepared by reducing $\mathrm{Ag}^{+}$with hydroxylamine hydrochloride [5]. Thus, $17 \mathrm{mg} \mathrm{AgNO}_{3}$ were solved in $90 \mathrm{ml}$ distilled water. $17 \mathrm{mg}$ $\mathrm{H}_{2} \mathrm{NOH} \times \mathrm{HCl}$ and $0.25 \mathrm{ml} \mathrm{NaOH}(2 \mathrm{M})$ were solved in to $10 \mathrm{ml}$ distilled water and then added rapidly to the silver nitrate solution under vigorous stirring. The final $\mathrm{pH}$ of the colloidal solution can be adjusted by varying the amount of $\mathrm{NaOH}$ added to the $\mathrm{H}_{2} \mathrm{NOH}$ solution, or adding diluted $\mathrm{HCl}$ solutions to the prepared colloid.

\section{Computational details}

For the proper understanding of the spectra, DFT especially hybrid functional methods are used to determine the electronic structure of molecules. For the assignment of the marker bands from SERS spectra, DFT calculations were assessed using B3LYP hybrid exchange-correlation functional and the 6-31G(d) basis set. The gas-phase optimized geometry of the neutral form of AZT and the optimized one in water as solvent were obtained, according to the Polarizable Continuum Model (PCM) solvation model.

$\mathrm{p} K_{\mathrm{a}}$ values of AZT were calculated using ACDLABS 12.0 (Advanced Chemistry Development, Canada) software. Both species, neutral and deprotonated, are present, with equal contributions, for the $\mathrm{p} K_{\mathrm{a}}$ value 9.55. In a previous study, Checa et al. [1] predicted a $\mathrm{p} K_{\mathrm{a}}$ value of 9.78 .

\section{Results and discussion}

Raman and SERS spectroscopies provide important structural information making them a powerful molecular investigation tool. For SERS measurements, a colloidal substrate at $\mathrm{pH}=7$ was prepared, where the neutral molecular form of AZT is predominantly present (in the 5-9 $\mathrm{pH}$ values interval), and also an alkaline one at $\mathrm{pH}=12$, where the deprotonated molecular form is dominant.

As shown in Fig. 1, the most intense bands of zidovudine are shown at $1648 \mathrm{~cm}^{-1}$ for the deprotonated species ( $\mathrm{pH} \mathrm{12)} \mathrm{and} \mathrm{at} 1643 \mathrm{~cm}^{-1}$ for the neutral zidovudine species ( $\mathrm{pH} 7$ ), assigned to the symmetric stretching vibration of the $\mathrm{C}=\mathrm{C}$ bond of the pyrimidine ring. Another marker band for zidovudine is that 




Fig. 1. SERS spectra of $10^{-5} \mathrm{M}$ zidovudine at $\mathrm{pH}=7$ (top) and $\mathrm{pH}=12$ (bottom).

corresponding to the breathing vibration of the pyrimidine ring, shown at 789 and at $793 \mathrm{~cm}^{-1}$ for the deprotonated species and for the neutral one, respectively.

Comparing the two SERS spectra, the most notable shifting occurs for the band at $1588 \mathrm{~cm}^{-1}$ in the case of the deprotonated form of zidovudine, to $1575 \mathrm{~cm}^{-1}$ in the spectrum of the neutral molecular species. This band has no correspondent in the FT-Raman spectrum. In our opinion, this band is due to the $\mathrm{C}=\mathrm{O}$ stretching vibrations of the pyrimidine ring, strongly shifted to lower wavenumbers, due to the interaction of oxygen with the silver surface. Furthermore, a characteristic band of zidovudine is the $\mathrm{N} \equiv \mathrm{N}$ stretching vibration of the azide group present in the SERS spectrum at $2114 \mathrm{~cm}^{-1}$.

\subsection{Adsorption geometry of zidovudine}

MEP is widely used as a reactivity map, displaying most probable regions for the electrophilic attack of charged point-like reagents on organic molecules [3]. Also, it is a useful tool in determining the adsorption geometry on the silver surface, considering also several marker bands. The MEP distribution of AZT molecule obtained from DFT calculations (Fig. 2) shows that the negative charge is located mainly on the oxygen atoms, thus, it is supposed that AZT adsorbs to the silver surface by the oxygen atoms of the pyrimidine ring. This supposition is sustained by the strong shifting of the $\mathrm{C}=\mathrm{O}$ band of the pyrimidine ring. The presence of the $\mathrm{C}=\mathrm{O}$ stretchings as well as of the breathing vibration of the pyrimidine ring as intense bands indicates a predominant perpendicular adsorption of the pyrimidine ring in respect to the silver surface, as can be deduced from the SERS selection rules $[6,8]$. Also, the enhancement of the $=\mathrm{C}-\mathrm{H}$ stretching of the pyrimidine ring at $3073 \mathrm{~cm}^{-1}$ sustains the predominant 


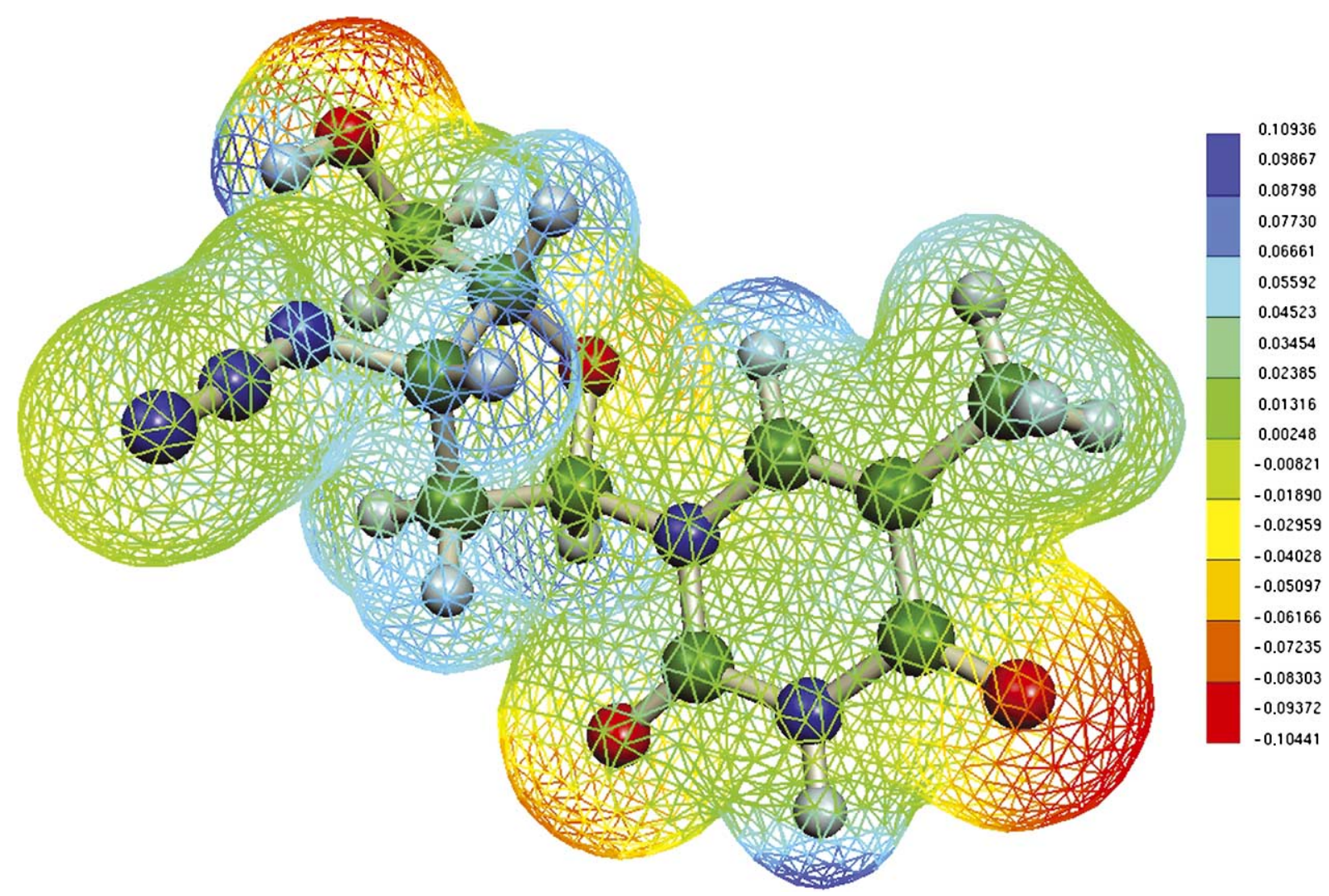

Fig. 2. B3LYP/6-31G(d) calculated 3D electrostatic potential of zidovudine (a.u) mapped onto the electronic density isosurface of 0.2 a.u. (Colors are visible in the online version of the article; http://dx.doi.org/10.3233/SPE-2011-0544.)

perpendicular orientation of the pyrimidine ring. A predominant parallel orientation of the oxalate ring, with respect to the silver surface is proposed, due to the weak SERS bands associated with this ring.

\section{Conclusions}

The calculated $\mathrm{p} K_{\mathrm{a}}$ value for AZT is 9.55 and the deprotonation of the molecule occurs at the imide nitrogen of the pyrimidine ring.

Normal modes of vibration including Raman intensities were calculated at B3LYP/6-31G(d) level of theory on the optimized structures of neutral and deprotonated molecular forms of zidovudine.

The characteristic bands of the neutral and deprotonated molecular forms were evidenced in the SERS spectra recorded at $\mathrm{pH} 7$ and $\mathrm{pH} 12$, respectively.

The pyrimidine ring appeared to have a perpendicular orientation on the silver surface, while the oxalate ring seems to be adsorbed predominantly parallel to the surface.

\section{Acknowledgements}

This work was supported by CNCS-UEFISCDI, project number PN II-RU TE_323/2010. Financial support from the Sectoral Operational Programme for Human Resources Development 2007-2013, co- 
financed by the European Social Fund, under the project number POSDRU/107/1.5/S/76841 is highly acknowledged by N.E.M.

\section{References}

[1] A. Checa, V. Gonzalez Soto, S. Hernandez-Cassou and J. Saurina, Fast determination of $\mathrm{p} K_{\mathrm{a}}$ values of reverse transcriptase inhibitor drugs for AIDS treatment by using $\mathrm{pH}$-gradient flow-injection analysis and multivariate curve resolution, Analytical Chimica Acta 554 (2005), 177-183.

[2] F. Cheng, L. Selvam and F. Wang, Blue shifted intramolecular C-H. . .O improper hydrogen bonds in conformers of zidovudine, Chemical Physics Letters 493 (2010), 358-363.

[3] V. Chiş, M.M. Venter, N. Leopold and O. Cozar, Raman, surface-enhanced Raman scattering and DFT study of para-nitroaniline, Vibrational Spectroscopy 48 (2008), 210-214.

[4] H. Kruszewska, U. Chmielowiec, E. Bednarek, J. Witowska-Jarosz, J.C. Dobrowolski and A. Misicka, Spectroscopic identification of AZT derivative obtained from biotransformation of AZT by Stenotrophomonas maltophilia, Journal of Molecular Structure 651-653 (2003), 645-650.

[5] N. Leopold and B. Lendl, A new method for fast preparation of highly Surface-Enhanced Raman Scattering (SERS) active silver colloids at room temperature by reduction of silver nitrate with hydroxylamine hydrochloride, Journal of Physical Chemistry B 107 (2003), 5723-5727.

[6] M. Moskovits, Surface-enhanced spectroscopy, Reviews on Modern Physics 57 (1985), 783-826.

[7] U.Y. Nayak, S. Gopal, S. Mutalik, A.K. Ranjith, M.S. Reddy, P. Gupta and N. Udupa, Glutaraldehyde cross-linked chitosan microspheres for controlled delivery of zidovudine, Journal of Microencapsulation 26 (2009), 214-222.

[8] A. Otto, I. Mrozek, H. Grabhorn and W. Akemann, Surface-enhanced Raman scattering, Journal of Physics: Condensed Matter 4 (1992), 1143-1212.

[9] A. Peepliwal, S.D. Vyawahare and C.G. Bonde, A quantitative analysis of zidovudine containing formulation by FT-IR and UV spectroscopy, Analytical Methods 2 (2010), 1756-1763.

[10] G. Ramachandran, A.K. Hemanthkumar, V. Kumaraswami and S. Swaminathan, A simple and rapid liquid chromatography method for simultaneous determination of zidovudine and nevirapine in plasma, Journal of Chromatography B $\mathbf{8 4 3}$ (2006), 339-344.

[11] M.A. Raviolo, P.A.M. Williams, S.B. Etcheverry, O.E. Piro, E.E. Castellano, M.S. Gualdesi and M.C. Briñón, Synthesis, molecular structure and physicochemical properties of bis $\left(3^{\prime}\right.$-azido- $3^{\prime}$-deoxythymidin- $5^{\prime}$-yl $)$ carbonate, Journal of Molecular Structure 970 (2010), 59-65.

[12] L. Rivas, S. Sanchez-Cortes and J.V. Garcia-Ramos, Conformational study of AZT in aqueous solution and adsorbed on a silver surface by means of Raman spectroscopy, Journal of Raman Spectroscopy 33 (2001), 6-9.

[13] L. Rivas, S. Sanchez-Cortes and J.V. Garcia-Ramos, Raman structural study of thymine and its 20-deoxy-ribosyl derivatives in solid state, aqueous solution and when adsorbed on silver nanoparticles, Physical Chemistry Chemical Physic 4 (2002), 1943-1948.

[14] F.X. Zhou, Z.K. Liao, J. Dai, J. Xiong, C.H. Xie, Z.G. Luo, S.Q. Liu and Y.F. Zhou, Radiosensitization effect of zidovudine on human malignant glioma cells, Biochemical and Biophysical Research Communications 354 (2007), 351-356. 


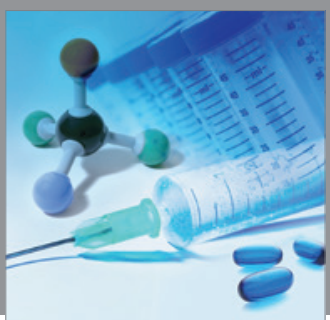

International Journal of

Medicinal Chemistry



Carbohydrate Chemistry

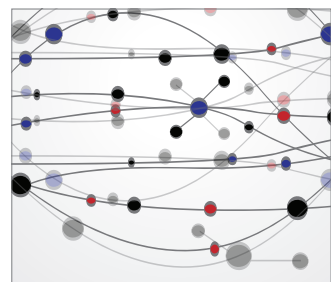

The Scientific World Journal

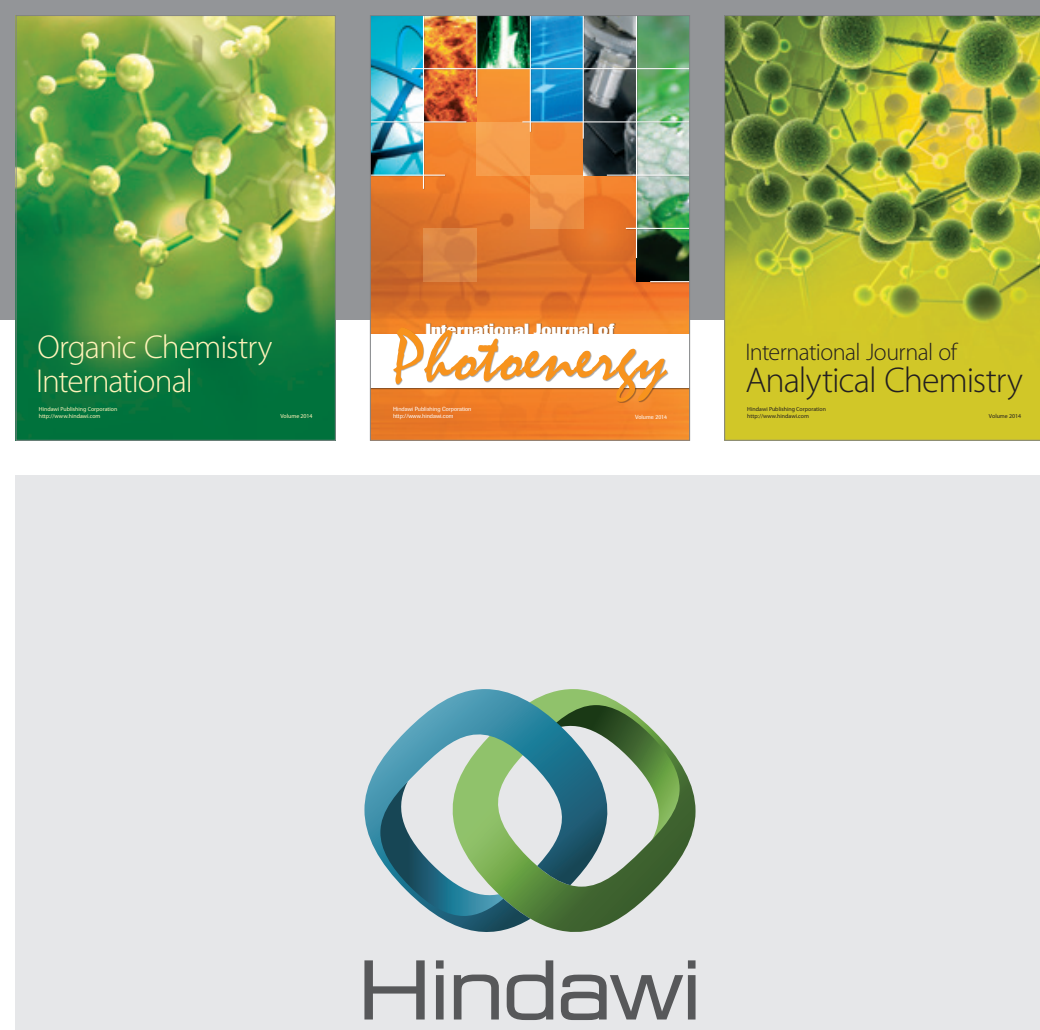

Submit your manuscripts at

http://www.hindawi.com


Journal of

Applied Chemistry
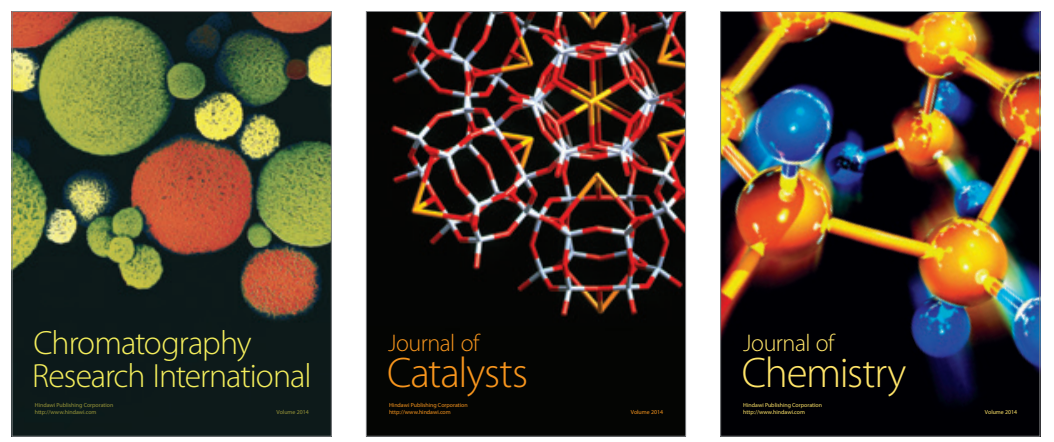
